\title{
Using Copy Number Alterations to Identify New Therapeutic Targets for Bladder Carcinoma
}

\author{
Donatella Conconi ${ }^{1}$, Elena Sala ${ }^{2}$, Giorgio Bovo ${ }^{3}$, Guido Strada ${ }^{4}$, Leda Dalprà ${ }^{1,2}$, \\ Marialuisa Lavitrano ${ }^{1}$ and Angela Bentivegna ${ }^{1, *}$ \\ 1 School of Medicine and Surgery, University of Milano-Bicocca, 20900 Monza, Italy; \\ donatella.conconi@unimib.it (D.C.); leda.dalpra@unimib.it (L.D.); marialuisa.lavitrano@unimib.it (M.L.) \\ 2 Medical Genetics Laboratory, San Gerardo Hospital, 20900 Monza, Italy; elena.sala@hsgerardo.org \\ 3 Department of Pathology, San Gerardo Hospital, 20900 Monza, Italy; g.bovo@hsgerardo.org \\ 4 Urology Division, Bassini Icp Hospital, 20092 Cinisello Balsamo, Italy; guido.strada@icp.mi.it \\ * Correspondence: angela.bentivegna@unimib.it; Tel.: +39-026-448-8133; Fax: +39-026-448-8253
}

Academic Editor: William Chi-shing Cho

Received: 11 January 2016; Accepted: 17 February 2016; Published: 24 February 2016

\begin{abstract}
Bladder cancer represents the ninth most widespread malignancy throughout the world. It is characterized by the presence of two different clinical and prognostic subtypes: non-muscle-invasive bladder cancers (NMIBCs) and muscle-invasive bladder cancers (MIBCs). MIBCs have a poor outcome with a common progression to metastasis. Despite improvements in knowledge, treatment has not advanced significantly in recent years, with the absence of new therapeutic targets. Because of the limitations of current therapeutic options, the greater challenge will be to identify biomarkers for clinical application. For this reason, we compared our array comparative genomic hybridization (array-CGH) results with those reported in literature for invasive bladder tumors and, in particular, we focused on the evaluation of copy number alterations (CNAs) present in biopsies and retained in the corresponding cancer stem cell (CSC) subpopulations that should be the main target of therapy. According to our data, CCNE1, MYC, MDM2 and PPARG genes could be interesting therapeutic targets for bladder CSC subpopulations. Surprisingly, HER2 copy number gains are not retained in bladder CSCs, making the gene-targeted therapy less interesting than the others. These results provide precious advice for further study on bladder therapy; however, the clinical importance of these results should be explored.
\end{abstract}

Keywords: bladder cancer; transitional cell carcinomas; therapeutic targets; cancer stem cells; copy number alterations

\section{Introduction}

Bladder cancer represents the ninth most widespread malignancy with 429,000 new cases and about 165,000 fatalities in 2012 ( $2 \%$ of the total number of cancer deaths). It occurs in men more than in women, with a sex ratio of 3.5 [1]. More than $90 \%$ of these tumors are transitional cell carcinomas (TCC, also urothelial carcinoma). This tumor is characterized by the presence of two different clinical and prognostic subtypes: non-muscle-invasive bladder cancers (NMIBCs) and muscle-invasive bladder cancers (MIBCs). At diagnosis the majority are non-muscle-invasive, papillary and low grade. NMIBC has the highest recurrence rate $(50 \%-80 \%)$ of any carcinoma and, consequently, this is the most expensive carcinoma per patient between diagnosis and death [2] because of repetitive and costly follow-up. However, the prognosis is usually good, with only $10 \%-15 \%$ progressing towards invasion while the five-year survival rate is $90 \%$. On the contrary, about $20 \%$ of cancers present muscle invasion 
at diagnosis, an unfavorable outcome with a survival rate after five years below 50\% [3] and common progression to metastasis.

For this reason, two different potential pathways have been proposed. The onset of NMIBCs may be derived via simple hyperplasia and minimal dysplasia through the loss of heterozygosity of chromosome 9 and activating mutations of FGFR3, PIK3CA and STAG2. Invasive carcinomas could be due to TP53 mutation in addition to chromosome 9 deletions, but generally without FGFR3 mutations via flat dysplasia and carcinoma in situ [4].

A recent study has combined statistical analysis and computational modeling to identify co-occurrence and mutual exclusivity of genes involved in bladder cancer [5]. Authors confirmed that FGFR 3 and PIK3CA mutations along with CDKN2A deletions are more associated with the noninvasive pathway, whereas EGFR, E2F3 amplifications and TP53 mutations characterized the invasive pathway.

Despite improvements in knowledge, treatment has not advanced significantly in recent years, with the absence of new therapeutic targets. NMIBC treatment options include: removing the tumor(s) via transurethral resection with fulguration, eventually followed by instillation of intravesical chemotherapy and possibly periodic intravesical instillations of bacillus Calmette-Guérin for high risk of recurrent tumors [6]. Standard treatment for patients with MIBCs is either neoadjuvant multiagent cisplatin-based chemotherapy and then radical cystectomy and urinary deviation, or radiation therapy associated with chemotherapy [6]. Because of the limitations of current therapeutic options, the greater challenge will be to identify biomarkers for clinical application.

We have been studying bladder cancer genetics for many years. We firstly reported the isolation and biological characterization of putative bladder cancer stem cell (CSC) populations from primary TCCs [7]. CSCs expand as clonally derived spheres (urospheres) with extensive proliferation and self-renewal capabilities. These cells showed positivity for stem cell markers and they could differentiate in the presence of serum. Cytogenetic data indicated an enrichment of hypo- or near-diploid cells, without the complexity of fresh tumors. Subsequently, we drew a comparison between the results of the UroVysion test executed on freshly isolated nuclei and on formalin-fixed paraffin-embedded tissues from 22 TCCs and we found no significant differences. Then, from the comparison between array comparative genomic hybridization (array-CGH) findings and the specific chromosomal data of the UroVysion test, we proved that it is still recommended to apply these two synergistic techniques, as the former is able to detect genome-wide alterations, but the second can preserve the characteristics of the individual cells [8].

However, our most interesting study concerned the comparison between array-CGH profiles of CSCs and the primary biopsy, to evaluate if differences in the genomic signature already exist in the initial steps of low grade and high grade tumors [9]. We found that CSCs isolated from low grade biopsies are highly rearranged compared to the primary biopsy, with an incommensurate number of genomic losses. This seems to be an essential characteristic which diversifies the two types of tumor, not the result of alterations occurring by chance in culture. Our approach allowed us to show that the genomic profile of low grade tumors differs from high grade tumors also in the initial steps of tumorigenesis; furthermore, a subset of low grade tumors showed a major disposition to mislay genomic regions.

These results provide precious information on bladder carcinogenesis and may be useful for the identification of personalized therapy and of potential targetable biomarkers.

\section{Results and Discussion}

In this work, we compared our array-CGH results with those reported in literature for invasive bladder tumors (Table 1); in particular, we focused on the comparison of selected genes identified by the Cancer Genome Atlas analysis $[10,11]$ to be the most significant in this type of tumor. Our approach could lead to detecting potential therapeutic targets through the evaluation of copy number alterations (CNAs) both in biopsies and in the corresponding isolated CSC subpopulations. For example, CCNE1 (cyclin E1) gene amplification has been reported in 9\%-12\% (Table 1) of bladder cancer, but in our 
cases a higher percentage of copy number gain in biopsies (25\%) and especially in CSC subpopulations $(60 \%)$ occurred. The presence of gain in more than half of CSC samples made us speculate that it might be considered an interesting therapeutic target. Currently, amplification of CCNE1 is considered a well-defined target in high grade serous ovarian cancer [12]; however, in bladder cancer it was only identified as a potential prognostic marker [13].

MYC oncogene amplification has been reported in bladder cancers (13\%, Table 1): our results showed the same percentage of mosaic gain in invasive biopsies $(12.5 \%)$ whereas a higher percentage of mosaic gain $(40 \%)$ was found in the corresponding CSC subpopulations. Recently, the effect of intravesical instillation of $M Y C$ inhibitor on orthotropic bladder tumor growth has been reported [14], confirming its role as a promising target for bladder cancer therapy, even if there are no current clinical trials (http://clinicaltrials.gov; https:/ / www.clinicaltrialsregister.eu).

Also, $M D M 2$, a negative regulator of tumor suppressor $\mathrm{p} 53$, has been reported as a potential therapeutic target in urothelial carcinoma [15] and its amplification was found in $9 \%$ of invasive tumors (Table 1). Interestingly, our biopsies showed no amplifications, but CSC subpopulations had a $40 \%$ copy number gain, proposing a possible use of MDM2 inhibitors, which are in current clinical trials, in bladder cancer therapy [16].

Human epidermal growth factor receptor 2 (HER2) overexpression is a target of anti-HER2 therapies for amplified breast cancer. Our array-CGH results showed a higher percentage of copy number gain both in low grade non-infiltrating (LGNI) and high grade infiltrating (HGIN) tumors with respect to published data ( $30 \%$ and $37.5 \%$, respectively, versus $7 \%$ and $5 \%$, Table 1 ). Recent works have evaluated the role of $H E R 2$ in bladder cancer and also several trials are currently investigating the possible benefit of targeted therapies for patients [17].

For this reason, we decided to substantiate these results on another set of tumors with the fluorescence in situ hybridization (FISH) technique (Figure 1). We considered samples positive for HER 2 amplification that showed more than $10 \%$ of cells with an HER2/CEP17 (centromere 17) ratio higher than two or with more than six HER2 signals per nucleus independent of CEP17 signals [18]. We found that $66.7 \%$ of tumors (both LGNI and HGIN) were positive for HER2 amplification (Table 2). This disparity with array-CGH data could be explained by the peculiarity of the two techniques and the different information provided by them, as already reported in our previous paper [8].

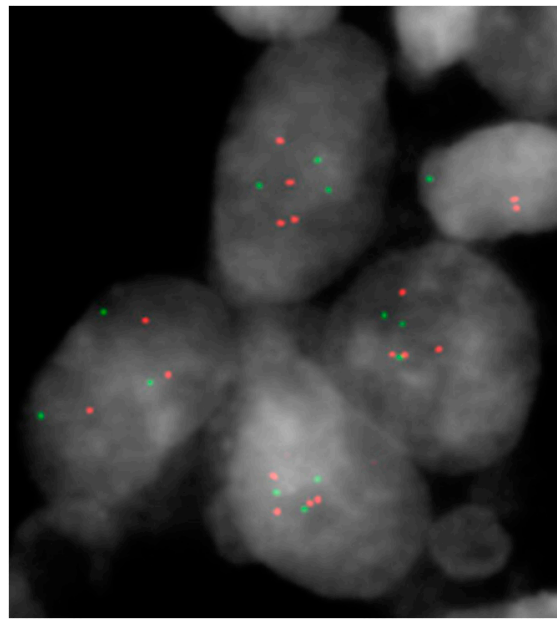

(a)

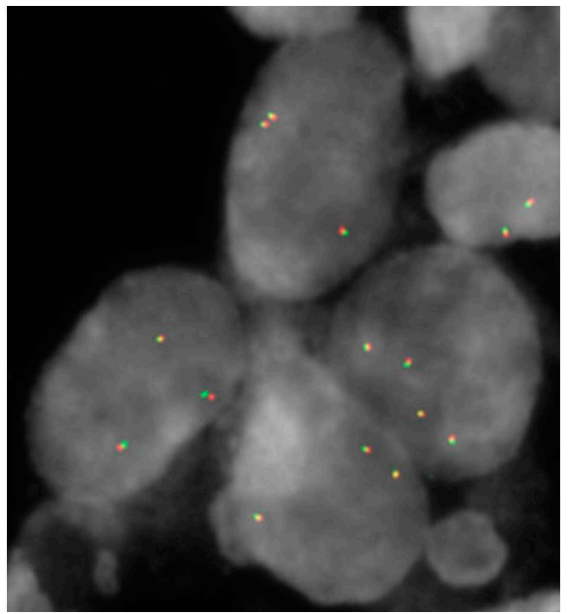

(b)

Figure 1. Examples of fluorescence in situ hybridization (FISH) analysis on formalin-fixed, paraffin-embedded (FFPE) tissues on the same tumoral area (case 27): (a) Her-2/Neu (17q12)/SE17 (SE: satellite enumeration) probe (human epidermal growth factor receptor 2 (HER2) red, centromere 17green); (b) PPAR $\gamma$ Break probe. Yellow dots represent the fusion of green and red signals of the dual color split probe. 
Table 1. Array comparative genomic hybridization (array-CGH) results. Comparison with literature.

\begin{tabular}{|c|c|c|c|c|c|c|c|c|c|c|c|c|}
\hline \multirow{2}{*}{ Genes } & \multicolumn{2}{|c|}{ Nature [10] } & \multicolumn{2}{|c|}{ Clin Cancer Res [11] } & \multicolumn{2}{|c|}{ Biopsies } & \multicolumn{2}{|c|}{ CSC Subpopulation } & \multicolumn{2}{|c|}{ Biopsies } & \multicolumn{2}{|c|}{ CSC Subpopulation } \\
\hline & $\begin{array}{l}\% \text { Loss } \\
\text { HGIN }\end{array}$ & $\begin{array}{l}\% \text { Gain } \\
\text { HGIN }\end{array}$ & $\begin{array}{l}\text { \% Loss } \\
\text { IN }\end{array}$ & $\begin{array}{c}\text { \% Gain } \\
\text { IN }\end{array}$ & $\begin{array}{c}\text { \% Loss } \\
\text { LGNI }\end{array}$ & $\begin{array}{l}\% \text { Loss } \\
\text { HGIN }\end{array}$ & $\begin{array}{c}\text { \% Loss } \\
\text { LGNI }\end{array}$ & $\begin{array}{l}\% \text { Loss } \\
\text { HGIN }\end{array}$ & $\begin{array}{c}\text { \% Gain } \\
\text { LGNI }\end{array}$ & $\begin{array}{l}\% \text { Gain } \\
\text { HGIN }\end{array}$ & $\begin{array}{c}\% \text { Gain } \\
\text { LGNI }\end{array}$ & $\begin{array}{l}\text { \% Gain } \\
\text { HGIN }\end{array}$ \\
\hline CCND1* & - & $10 \mathrm{~A}^{\circ}$ & - & $11 \mathrm{~A}$ & - & - & $10 \mathrm{M}$ & $20 \mathrm{M}$ & $\begin{array}{l}20 \mathrm{~A} \\
30 \mathrm{M}\end{array}$ & $\begin{array}{c}12.5 \mathrm{~A} \\
12.5 \mathrm{NM} \\
12.5 \mathrm{M}\end{array}$ & $\begin{array}{l}40 \mathrm{~A} \\
20 \mathrm{M}\end{array}$ & $\begin{array}{c}20 \mathrm{~A} \\
20 \mathrm{NM}\end{array}$ \\
\hline E2F3/SOX4 & - & $20 \mathrm{~A}^{\circ}$ & - & $18 \mathrm{~A}$ & - & - & - & - & - & $12.5 \mathrm{NM}$ & - & $20 \mathrm{NM}$ \\
\hline EGFR & - & $11 \mathrm{~A}^{\circ}$ & - & $7 \mathrm{~A}$ & - & - & $10 \mathrm{M}$ & - & $\begin{array}{c}20 \mathrm{NM} \\
10 \mathrm{M}\end{array}$ & $12.5 \mathrm{M}$ & $30 \mathrm{NM}$ & $20 \mathrm{M}$ \\
\hline PPARG & - & $17 \mathrm{~A}^{\circ}$ & - & $14 \mathrm{~A}$ & - & - & - & - & - & $\begin{array}{c}12.5 \mathrm{~A} \\
25 \mathrm{NM} \\
25 \mathrm{M}\end{array}$ & - & $40 \mathrm{M}$ \\
\hline PVRL4* & - & $19 \mathrm{~A}^{\circ}$ & - & $17 \mathrm{~A}$ & - & - & $\begin{array}{c}10 \mathrm{CL} \\
20 \mathrm{NM}\end{array}$ & $20 \mathrm{NM}$ & $\begin{array}{c}10 \mathrm{NM} \\
10 \mathrm{M}\end{array}$ & $75 \mathrm{M}$ & $\begin{array}{c}30 \mathrm{NM} \\
10 \mathrm{M}\end{array}$ & $40 \mathrm{M}$ \\
\hline YWHAZ* & - & $22 \mathrm{~A}^{\circ}$ & - & $22 \mathrm{~A}$ & - & - & - & - & $10 \mathrm{NM}$ & $25 \mathrm{NM}$ & $10 \mathrm{NM}$ & $40 \mathrm{NM}$ \\
\hline MDM2 & - & $9 \mathrm{~A}^{\circ}$ & - & $9 \mathrm{~A}$ & - & - & $10 \mathrm{M}$ & - & $20 \mathrm{M}$ & - & $10 \mathrm{M}$ & $\begin{array}{c}20 \mathrm{NM} \\
20 \mathrm{M}\end{array}$ \\
\hline HER2 & - & $7 \mathrm{~A}^{\circ}$ & - & $5 \mathrm{~A}$ & - & - & $20 \mathrm{M}$ & - & $\begin{array}{c}20 \mathrm{M} \\
10 \mathrm{NM}\end{array}$ & $37.5 \mathrm{M}$ & $10 \mathrm{M}$ & - \\
\hline YAP1 & - & $4 \mathrm{~A}^{\circ}$ & - & $\mathrm{ni}$ & $10 \mathrm{M}$ & $12.5 \mathrm{M}$ & - & - & - & $12.5 \mathrm{M}$ & - & $25 \mathrm{M}$ \\
\hline CCNE1 & - & $12 \mathrm{~A}^{\circ}$ & - & $9 \mathrm{~A}$ & - & $12.5 \mathrm{M}$ & - & - & - & $25 \mathrm{M}$ & - & $60 \mathrm{M}$ \\
\hline$M Y C$ & - & $13 \mathrm{~A}^{\circ}$ & - & $13 \mathrm{~A}$ & - & $12.5 \mathrm{M}$ & $\begin{array}{c}10 \mathrm{NM} \\
10 \mathrm{M}\end{array}$ & - & - & $12.5 \mathrm{M}$ & $10 \mathrm{M}$ & $40 \mathrm{M}$ \\
\hline FGFR3 * & - & $3 \mathrm{~A}^{\circ}$ & - & $4 \mathrm{~A}$ & - & $12.5 \mathrm{M}$ & $20 \mathrm{M}$ & $20 \mathrm{M}$ & $\begin{array}{l}10 \mathrm{~A} \\
40 \mathrm{M} \\
\end{array}$ & $12.5 \mathrm{M}$ & $\begin{array}{l}10 \mathrm{~A} \\
10 \mathrm{M} \\
\end{array}$ & $20 \mathrm{M}$ \\
\hline MYCL1* & - & $6 \mathrm{~A}^{\circ}$ & & $6 \mathrm{~A}$ & - & - & - & - & $10 \mathrm{M}$ & $12.5 \mathrm{M}$ & $10 \mathrm{NM}$ & - \\
\hline$B C L 2 L 1$ & - & $11 \mathrm{~A}^{\circ}$ & - & $10 \mathrm{~A}$ & - & - & $\begin{array}{c}10 \mathrm{CL} \\
20 \mathrm{NM}\end{array}$ & - & $50 \mathrm{M}$ & $\begin{array}{c}12.5 \mathrm{~A} \\
12.5 \mathrm{NM} \\
37.5 \mathrm{M}\end{array}$ & $\begin{array}{c}10 \mathrm{~A} \\
20 \mathrm{NM} \\
10 \mathrm{M}\end{array}$ & $20 \mathrm{NM}$ \\
\hline BEND3 * & - & ni & - & $3 \mathrm{~A}$ & - & $37.5 \mathrm{NM}$ & - & - & $10 \mathrm{M}$ & $25 \mathrm{NM}$ & $\begin{array}{c}20 \mathrm{NM} \\
10 \mathrm{M}\end{array}$ & - \\
\hline BIRC3 & - & ni & - & $4 \mathrm{~A}$ & $20 \mathrm{M}$ & $37.5 \mathrm{M}$ & $\begin{array}{c}10 \mathrm{NM} \\
10 \mathrm{M}\end{array}$ & $20 \mathrm{M}$ & - & - & $10 \mathrm{M}$ & $\begin{array}{c}20 \mathrm{NM} \\
20 \mathrm{M}\end{array}$ \\
\hline
\end{tabular}


Table 1. Cont

\begin{tabular}{|c|c|c|c|c|c|c|c|c|c|c|c|c|}
\hline \multirow{2}{*}{ Genes } & \multicolumn{2}{|c|}{ Nature [10] } & \multicolumn{2}{|c|}{ Clin Cancer Res [11] } & \multicolumn{2}{|c|}{ Biopsies } & \multicolumn{2}{|c|}{ CSC Subpopulation } & \multicolumn{2}{|c|}{ Biopsies } & \multicolumn{2}{|c|}{ CSC Subpopulation } \\
\hline & $\begin{array}{l}\% \text { Loss } \\
\text { HGIN }\end{array}$ & $\begin{array}{l}\text { \% Gain } \\
\text { HGIN }\end{array}$ & $\begin{array}{l}\text { \% Loss } \\
\text { IN }\end{array}$ & $\begin{array}{l}\text { \% Gain } \\
\text { IN }\end{array}$ & $\begin{array}{c}\text { \% Loss } \\
\text { LGNI }\end{array}$ & $\begin{array}{c}\text { \% Loss } \\
\text { HGIN }\end{array}$ & $\begin{array}{l}\text { \% Loss } \\
\text { LGNI }\end{array}$ & $\begin{array}{c}\text { \% Loss } \\
\text { HGIN }\end{array}$ & $\begin{array}{c}\text { \% Gain } \\
\text { LGNI }\end{array}$ & $\begin{array}{c}\text { \% Gain } \\
\text { HGIN }\end{array}$ & $\begin{array}{c}\text { \% Gain } \\
\text { LGNI }\end{array}$ & $\begin{array}{l}\text { \% Gain } \\
\text { HGIN }\end{array}$ \\
\hline GDI2 * & - & $\mathrm{ni}$ & - & $9 \mathrm{~A}$ & $30 \mathrm{M}$ & $12.5 \mathrm{M}$ & $10 \mathrm{M}$ & & $\begin{array}{c}10 \mathrm{NM} \\
10 \mathrm{M}\end{array}$ & $25 \mathrm{M}$ & $10 \mathrm{NM}$ & $60 \mathrm{M}$ \\
\hline PRKCI & - & ni & & $4 \mathrm{~A}$ & - & - & - & - & - & $12.5 \mathrm{M}$ & - & - \\
\hline SOX4 & - & $\mathrm{ni}$ & & $18 \mathrm{~A}$ & - & - & - & - & - & $12.5 \mathrm{NM}$ & - & $20 \mathrm{NM}$ \\
\hline CDKN2A & $47 \mathrm{D}^{\circ}$ & - & $43 \mathrm{D}$ & & $\begin{array}{l}30 \mathrm{CL} \\
10 \mathrm{M}\end{array}$ & $\begin{array}{c}12.5 \mathrm{CL} \\
12.5 \mathrm{NM} \\
25 \mathrm{M}\end{array}$ & $30 \mathrm{CL}$ & $\begin{array}{c}20 \mathrm{CL} \\
40 \mathrm{M}\end{array}$ & - & - & - & - \\
\hline PTEN & $13 \mathrm{D}^{\circ}$ & - & $13 \mathrm{D}$ & - & - & - & - & - & $10 \mathrm{M}$ & - & - & $20 \mathrm{NM}$ \\
\hline NCOR1 & $25 \mathrm{D}^{\circ}$ & - & $24 \mathrm{D}$ & - & - & $25 \mathrm{M}$ & - & $20 \mathrm{M}$ & - & - & - & - \\
\hline CREBBP & $13 \mathrm{D}^{\circ}$ & - & $16 \mathrm{D}$ & - & $20 \mathrm{M}$ & $25 \mathrm{M}$ & $10 \mathrm{M}$ & - & - & $37.5 \mathrm{M}$ & - & - \\
\hline$R B 1$ & $14 \mathrm{D}^{\circ}$ & - & $17 \mathrm{D}$ & - & - & $12.5 \mathrm{M}$ & $40 \mathrm{M}$ & $20 \mathrm{M}$ & - & $12.5 \mathrm{M}$ & - & - \\
\hline ARID1A & ni & - & $5 \mathrm{D}$ & - & $10 \mathrm{M}$ & - & - & - & - & - & - & - \\
\hline FHIT & $\mathrm{ni}$ & - & $13 \mathrm{D}$ & - & $10 \mathrm{M}$ & $12.5 \mathrm{M}$ & $10 \mathrm{M}$ & $20 \mathrm{M}$ & - & $12.5 \mathrm{M}$ & - & - \\
\hline$I K Z F 2$ & $\mathrm{ni}$ & - & $15 \mathrm{D}$ & - & - & $50 \mathrm{M}$ & - & $20 \mathrm{M}$ & $10 \mathrm{M}$ & - & - & - \\
\hline$L R P 1 B$ & $\mathrm{ni}$ & - & $17 \mathrm{D}$ & - & - & $37.5 \mathrm{M}$ & - & $20 \mathrm{M}$ & - & - & - & - \\
\hline PDE4D & $\mathrm{ni}$ & - & $22 \mathrm{D}$ & - & - & $25 \mathrm{M}$ & - & $20 \mathrm{M}$ & - & - & - & - \\
\hline WWOX & ni & - & $15 \mathrm{D}$ & - & - & $12.5 \mathrm{M}$ & - & - & - & - & - & - \\
\hline
\end{tabular}

$\mathrm{A}^{\circ}$ : copy number $>3$; $\mathrm{D}^{\circ}$ : copy number < 1.5; A: amplification; CL: complete loss; M: mosaic; NM: non-mosaic; D: deletion; ni: no information; LGNI: low grade non-infiltrating; HGIN: high grade infiltrating; CSC: cancer stem cell; *: only one or two probes. 
Table 2. Fluorescence in situ hybridization (FISH) on formalin-fixed, paraffin-embedded (FFPE) tissues.

\begin{tabular}{|c|c|c|c|}
\hline HGIN & HER $2 \%$ of Amplified Cells & PPARG > Two Signals & $C D K N 2 A<$ Two Signals \\
\hline 19 & $22 \%$ & $\begin{array}{c}32 \% \\
\mathrm{~m}=2.36\end{array}$ & $\begin{array}{c}100 \% \\
m=0.08\end{array}$ \\
\hline 20 & $6 \%$ & - & $\begin{array}{c}85 \% \\
\mathrm{~m}=1.02\end{array}$ \\
\hline 21 & $84 \%$ & $\begin{array}{l}56.4 \% \\
\mathrm{~m}=3\end{array}$ & $\begin{array}{c}58 \% \\
\mathrm{~m}=1.19\end{array}$ \\
\hline 22 & $80 \%$ & $\begin{array}{c}50 \% \\
\mathrm{~m}=2.8\end{array}$ & $\begin{array}{c}6 \% \\
m=2.94\end{array}$ \\
\hline 23 & $30 \%$ & $\begin{array}{c}63.6 \% \\
m=2.97\end{array}$ & $\begin{array}{c}10 \% \\
m=2.27\end{array}$ \\
\hline 24 & $2.5 \%$ & $\begin{array}{c}48 \% \\
m=2.66\end{array}$ & - \\
\hline 25 & $3.3 \%$ & $\begin{array}{c}42 \% \\
\mathrm{~m}=2.4\end{array}$ & $\begin{array}{c}100 \% \\
\mathrm{~m}=0.02\end{array}$ \\
\hline 26 & $20 \%$ & $\begin{array}{c}28 \% \\
\mathrm{~m}=2.34\end{array}$ & $\begin{array}{c}7 \% \\
\mathrm{~m}=2.72\end{array}$ \\
\hline 27 & $37.5 \%$ & $\begin{array}{c}87.5 \% \\
m=3.7\end{array}$ & $\begin{array}{c}43 \% \\
m=1.83\end{array}$ \\
\hline LGNI & HER $2 \%$ of Amplified Cells & PPARG > Two Signals & $C D K N 2 A<$ Two Signals \\
\hline 28 & $8 \%$ & $\begin{array}{c}4 \% \\
\mathrm{~m}=2.02\end{array}$ & $\begin{array}{c}100 \% \\
m=0.17\end{array}$ \\
\hline 29 & $10 \%$ & - & $\begin{array}{c}61 \% \\
\mathrm{~m}=1.28\end{array}$ \\
\hline 30 & $13 \%$ & $\begin{array}{c}10 \% \\
\mathrm{~m}=1.6\end{array}$ & $\begin{array}{l}100 \% \\
\mathrm{~m}=0\end{array}$ \\
\hline 31 & $32 \%$ & $\begin{array}{c}2 \% \\
\mathrm{~m}=1.72\end{array}$ & $\begin{array}{c}58 \% \\
\mathrm{~m}=1.21\end{array}$ \\
\hline 32 & $14 \%$ & $\begin{array}{c}38 \% \\
\mathrm{~m}=2.52\end{array}$ & $\begin{array}{c}99 \% \\
\mathrm{~m}=0.03\end{array}$ \\
\hline 33 & $30 \%$ & $\begin{array}{c}4 \% \\
\mathrm{~m}=1.76\end{array}$ & - \\
\hline
\end{tabular}

Then our cohort of Italian patients showed a significant variance in HER2 amplification with respect to published data. In particular, The Cancer Genome Atlas Project identified 7\% amplification in 131 patients with high grade urothelial cancer [10] and other studies displayed a frequency of amplification ranging from $5 \%$ to $14 \%$, with the exception of $42 \%$ detected in the infrequent micropapillary histological variant [4]. However, a recent study on two different cohorts of patients proved a significant difference in frequencies of HER2 amplification between the Spanish and Greek patients, with 20\% and 4\%, respectively. These results suggest that HER2 amplification can change between populations and promote the hypothesis that etiologic heterogeneity can lead to these differences [19].

To evaluate if HER2 may be a therapeutic target in a subgroup of urothelial carcinomas, we analyzed the results obtained in the CSCs subpopulation. Comparison between biopsies and the corresponding CSCs showed that HER2 copy number gains are not retained in CSCs except for one case (Table 3). This result could suggest the hypothesis that HER2 amplification is not present in bladder cancer tumor-initiating cells. Moreover, the majority of HER2 amplifications detected in 
biopsies are as a mosaic (Table 3), so a low level of mosaicism amplification could be also in CSCs, but not detectable by the array-CGH technique.

Table 3. Array-CGH results. Comparison between biopsies and cancer stem cells.

\begin{tabular}{|c|c|c|c|c|c|c|c|}
\hline Histotype & $\begin{array}{c}\text { CASE } \\
\mathbf{n}^{\circ}\end{array}$ & \multicolumn{3}{|c|}{ Biopsies } & \multicolumn{3}{|c|}{ Cancer Stem Cells } \\
\hline \multirow{8}{*}{ LGNI } & 1 & disomy & disomy & disomy & disomy & disomy & disomy \\
\hline & 3 & disomy & disomy & disomy & mosaic loss & disomy & disomy \\
\hline & 4 & non mosaic gain & disomy & disomy & mosaic loss & disomy & disomy \\
\hline & 5 & mosaic gain & disomy & mosaic loss & mosaic gain & disomy & disomy \\
\hline & 7 & disomy & disomy & disomy & disomy & disomy & disomy \\
\hline & 8 & disomy & disomy & complete loss & disomy & disomy & complete loss \\
\hline & 9 & disomy & disomy & disomy & disomy & disomy & disomy \\
\hline & 10 & disomy & disomy & complete loss & disomy & disomy & complete loss \\
\hline \multirow{5}{*}{ HGIN } & 11 & disomy & non mosaic gain & mosaic loss & disomy & disomy & disomy \\
\hline & 12 & mosaic gain & mosaic gain & complete loss & disomy & mosaic gain & mosaic loss \\
\hline & 16 & disomy & amplification & disomy & - & - & - \\
\hline & 17 & disomy & disomy & disomy & - & - & - \\
\hline & 18 & mosaic gain & disomy & disomy & - & - & - \\
\hline
\end{tabular}

The most known gene involved in bladder cancer is CDKN2A. Our array-CGH results are in agreement with the literature, with a $40 \%$ loss in LGNI biopsies ( $30 \%$ complete loss) and $50 \%$ in HGIN biopsies (12.5\% complete loss); instead, FISH analysis performed on another set of tumor biopsies revealed a $100 \%$ loss in LGNI (5/5 cases) and $62.5 \%$ in HGIN samples (5/8 cases) with a median number of signals less than two (Table 2). CDKN2A loss was also maintained in CSC subpopulations ( $30 \%$ and $60 \%$ in LGNI and HGIN, respectively), denoting its important role in cancer onset and progression (Table 3).

Finally, PPARG results were analyzed. We reported PPARG amplification in three samples derived from the same patient with multifocal non-muscle-invasive bladder cancer, giving a novel proof on the PPARG role in onset and support of bladder cancer, and also a potential explanation for the monoclonal origin of multifocality [20].

PPARG amplification has been reported in $14 \%-17 \%$ of bladder cancer; our HGIN biopsies showed $12.5 \%$ amplification but also 50\% copy number gain (Table 1). FISH analysis performed on another set of tumor biopsies revealed a $20 \%$ of gain in LGNI (1/5) and $100 \%$ in HGIN samples (8/8) with a median number of signals greater than two (Table 2). PPARG gain was maintained in $40 \%$ of CSCs, only as mosaic gain (Table 3).

\section{Materials and Methods}

\subsection{Tumor Specimens}

TCC specimens were collected from 33 patients that underwent transurethral resection at a single center, as previously reported [9]. Staging and grading were done according to the World Health Organization Consensus Classification by a pathologist. Samples were classified as high or low grade (HG or LG) and muscle infiltrating or non-muscle-infiltrating (IN or NI) (see Tables 2 and 3). No patient has been treated before surgery.

This study was approved and founded by Direzione Generale Sanità Regione Lombardia and presented by General Director and ethic commitment of ICP Hospital Bassini (Cinisello Balsamo, Italy), as previously reported [9]. Written informed consent was obtained before tissue collection. 


\subsection{Array Comparative Genomic Hybridization (Array-CGH)}

Genomic DNA extraction, sample preparation, slide hybridization and analysis were performed using SurePrint G3 Human CGH Microarray 8x60K (Agilent Technologies, Santa Clara, CA, USA) following the manufacturer's recommendations. The arrays were scanned at $2-\mu \mathrm{m}$ resolution and analyzed using Feature Extraction v10.7 and Agilent Genomic Workbench v5.0 software (Agilent Technologies), as previously reported [9].

\subsection{Fluorescence in Situ Hybridization (FISH)}

FISH analysis on formalin-fixed, paraffin-embedded (FFPE) tissue sections was performed using Her-2/Neu (17q12)/SE17 probe (Kreatech Diagnostics, Amsterdam, The Netherlands), PPAR $\gamma$ (3p25) Break probe (Kreatech Diagnostics) and UroVysion bladder cancer kit (Vysis, Wiesbaden, Germany) according to the manufacturer's instructions. HER2 amplification is considered for samples that show more than $10 \%$ of cells with HER2/CEP17 ratio greater than two or with more than six HER2 signals per nucleus (ASCO international guidelines for breast cancer therapy) [18]. All digital images were captured using a Leitz microscope (Leica DM 5000B, Leica Microsystems GmbH, Wetzlar, Germany) equipped with a charge coupled device (CCD) camera (Leica Microsystems) and analyzed by means of Chromowin software (Tesi Imaging, Milano, Italy).

\section{Conclusions}

In conclusion, our approach allowed us to evaluate genes with copy number alterations in biopsies that are retained in the corresponding CSC subpopulations, which should be the main target of therapy. According to our data, CCNE1, MYC, MDM2 and PPARG genes could be interesting therapeutic targets for bladder CSC subpopulations in order to overcome the limitations of current therapeutic options. Surprisingly, HER2 copy number gains are not retained in bladder CSCs, making the gene-targeted therapy less interesting than the others. These results provide precious advice to further study on bladder cancer therapy; however, the clinical importance of these results should be explored.

Acknowledgments: This work was supported by Associazione Gianluca Strada Onlus. The funders had no role in study design, data collection and analysis, decision to publish, or preparation of the manuscript.

Author Contributions: Donatella Conconi concept and idea, performed the experiments, data analysis and interpretation, and writing the manuscript. Elena Sala interpretation/discussion of FISH analysis. Giorgio Bovo critical revision of FFPE Hematoxylin-eosin staining. Guido Strada patient selection. Leda Dalprà concept and idea, discussions, and critical revision/supervision. Marialuisa Lavitrano edited the manuscript. Angela Bentivegna concept and idea, data analysis and interpretation, and writing the manuscript.

Conflicts of Interest: The authors declare no conflict of interest.

\section{Abbreviations}

TCC: transitional cell carcinomas

NMIBC: non-muscle-invasive bladder cancer

MIBC: muscle-invasive bladder cancer

CSC: cancer stem cell

CGH: comparative genomic hybridization

CNA: copy number alteration

LGNI: low grade non-infiltrating

HGIN: high grade infiltrating

FISH: fluorescence in situ hybridization 


\section{References}

1. Ferlay, J.; Soerjomataram, I.; Dikshit, R.; Eser, S.; Mathers, C.; Rebelo, M.; Parkin, D.M.; Forman, D.; Bray, F. Cancer incidence and mortality worldwide: Sources, methods and major patterns in GLOBOCAN 2012. Int. J. Cancer 2015, 136, E359-E386. [CrossRef] [PubMed]

2. Cussenot, O.; Sighar, K.; Mohammed, M.; Hugonin, S.; Ondet, V.; Larre, S.; Lacave, R.; Roupret, M.; Cancel-Tassin, G. Detection of specific chromosomal aberrations in urine using BCA-1 (oligo-CGH-array) enhances diagnostic sensitivity and predicts the aggressiveness of non-muscle-invasive bladder transitional cell carcinoma. World J. Urol. 2014, 32, 551-557. [CrossRef] [PubMed]

3. Kawanishi, H.; Takahashi, T.; Ito, M.; Matsui, Y.; Watanabe, J.; Ito, N.; Kamoto, T.; Kadowaki, T.; Tsujimoto, G.; Imoto, I.; et al. Genetic analysis of multifocal superficial urothelial cancers by array-based comparative genomic hybridisation. Br. J. Cancer 2007, 97, 260-266. [CrossRef] [PubMed]

4. Knowles, M.A.; Hurst, C.D. Molecular biology of bladder cancer: New insights into pathogenesis and clinical diversity. Nat. Rev. Cancer 2015, 15, 25-41. [CrossRef] [PubMed]

5. Remy, E.; Rebouissou, S.; Chaouiya, C.; Zinovyev, A.; Radvanyi, F.; Calzone, L. A modeling approach to explain mutually exclusive and co-occurring genetic alterations in bladder tumorigenesis. Cancer Res. 2015, 75, 4042-4052. [CrossRef] [PubMed]

6. Physician Data Query Adult treatment editorial board. Bladder Cancer Treatment $\left(P D Q^{\circledR}\right)$ : Health Professional Version; National Cancer Institute (US): Bethesda, MD, USA, 2015.

7. Bentivegna, A.; Conconi, D.; Panzeri, E.; Sala, E.; Bovo, G.; Viganò, P.; Brunelli, S.; Bossi, M.; Tredici, G.; Strada, G.; et al. Biological heterogeneity of putative bladder cancer stem-like cell populations from human bladder transitional cell carcinoma samples. Cancer Sci. 2010, 101, 416-424. [CrossRef] [PubMed]

8. Panzeri, E.; Conconi, D.; Antolini, L.; Redaelli, S.; Valsecchi, M.G.; Bovo, G.; Pallotti, F.; Viganò, P.; Strada, G.; Dalprà, L.; et al. Chromosomal aberrations in bladder cancer: Fresh versus formalin fixed paraffin embedded tissue and targeted fish versus wide microarray-based CGH analysis. PLoS ONE 2011, 6, e24237. [CrossRef] [PubMed]

9. Conconi, D.; Panzeri, E.; Redaelli, S.; Bovo, G.; Viganò, P.; Strada, G.; Dalprà, L.; Bentivegna, A. Chromosomal imbalances in human bladder urothelial carcinoma: Similarities and differences between biopsy samples and cancer stem-like cells. BMC Cancer 2014, 14. [CrossRef] [PubMed]

10. Cancer Genome Atlas Research Network. Comprehensive molecular characterization of urothelial bladder carcinoma. Nature 2014, 507, 315-322.

11. Kim, J.; Akbani, R.; Creighton, C.J.; Lerner, S.P.; Weinstein, J.N.; Getz, G.; Kwiatkowski, D.J. Invasive bladder cancer: Genomic insights and therapeutic promise. Clin. Cancer Res. 2015, 21, 4514-4524. [CrossRef] [PubMed]

12. Etemadmoghadam, D.; Weir, B.A.; Au-Yeung, G.; Alsop, K.; Mitchell, G.; George, J.; Davis, S.; D'Andrea, A.D.; Simpson, K.; Hahn, W.C.; et al. Synthetic lethality between CCNE1 amplification and loss of BRCA1. Proc. Natl. Acad. Sci. USA 2013, 110, 19489-19494. [CrossRef] [PubMed]

13. Matsushita, R.; Seki, N.; Chiyomaru, T.; Inoguchi, S.; Ishihara, T.; Goto, Y.; Nishikawa, R.; Mataki, H.; Tatarano, S.; Itesako, T.; et al. Tumour-suppressive microRNA-144-5p directly targets CCNE1/2 as potential prognostic markers in bladder cancer. Br. J. Cancer 2015, 113, 282-289. [CrossRef] [PubMed]

14. Jeong, K.C.; Kim, K.T.; Seo, H.H.; Shin, S.P.; Ahn, K.O.; Ji, M.J.; Park, W.S.; Kim, I.H.; Lee, S.J.; Seo, H.K. Intravesical instillation of c-MYC inhibitor KSI-3716 suppresses orthotopic bladder tumor growth. J. Urol. 2014, 191, 510-518. [CrossRef] [PubMed]

15. Chekaluk, Y.; Wu, C.L.; Rosenberg, J.; Riester, M.; Dai, Q.; Lin, S.; Guo, Y.; McDougal, W.S.; Kwiatkowski, D.J. Identification of nine genomic regions of amplification in urothelial carcinoma, correlation with stage, and potential prognostic and therapeutic value. PLOS ONE 2013, 8, e60927. [CrossRef] [PubMed]

16. Zhao, Y.; Aguilar, A.; Bernard, D.; Wang, S. Small-molecule inhibitors of the MDM2-p53 protein-protein interaction (MDM2 inhibitors) in clinical trials for cancer treatment. J. Med. Chem. 2015, 58, 1038-1052. [CrossRef] [PubMed]

17. Tschui, J.; Vassella, E.; Bandi, N.; Baumgartner, U.; Genitsch, V.; Rotzer, D.; Seiler, R.; Thalmann, G.N.; Fleischmann, A. Morphological and molecular characteristics of HER2 amplified urothelial bladder cancer. Virchows Arch. 2015, 466, 703-710. [CrossRef] [PubMed] 
18. Wolff, A.C.; Hammond, M.E.; Hicks, D.G.; Dowsett, M.; McShane, L.M.; Allison, K.H.; Allred, D.C.; Bartlett, J.M.; Bilous, M.; Fitzgibbons, P.; et al. Recommendations for human epidermal growth factor receptor 2 testing in breast cancer: American society of clinical oncology/college of american pathologists clinical practice guideline update. J. Clin. Oncol. 2013, 31, 3997-4013. [CrossRef] [PubMed]

19. Bellmunt, J.; Werner, L.; Bamias, A.; Fay, A.P.; Park, R.S.; Riester, M.; Selvarajah, S.; Barletta, J.A.; Berman, D.M.; de Muga, S.; et al. HER2 as a target in invasive urothelial carcinoma. Cancer Med. 2015, 4, 844-852. [CrossRef] [PubMed]

20. Conconi, D.; Panzeri, E.; Redaelli, S.; Bovo, G.; Volante, M.; Viganò, P.; Strada, G.; Dalprà, L.; Bentivegna, A. DNA copy number alterations and PPARG amplification in a patient with multifocal bladder urothelial carcinoma. BMC Res. Notes 2012, 5. [CrossRef] [PubMed]

(C) 2016 by the authors; licensee MDPI, Basel, Switzerland. This article is an open access article distributed under the terms and conditions of the Creative Commons by Attribution (CC-BY) license (http://creativecommons.org/licenses/by/4.0/). 